\title{
Ceramide mediates nanovesicle shedding and cell death in response to phosphatidylinositol ether lipid analogs and perifosine
}

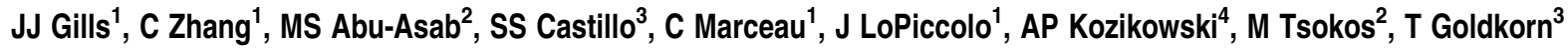 \\ and PA Dennis ${ }^{*, 1}$
}

Anticancer phospholipids that inhibit Akt such as the alkylphospholipid perifosine (Per) and phosphatidylinositol ether lipid analogs (PIAs) promote cellular detachment and apoptosis and have a similar cytotoxicity profile against cancer cell lines in the $\mathrm{NCl60}$ panel. While investigating the mechanism of Akt inhibition, we found that short-term incubation with these compounds induced rapid shedding of cellular nanovesicles containing EGFR, IGFR and p-Akt that occurred in vitro and in vivo, while prolonged incubation led to cell detachment and death that depended on sphingomyelinase-mediated generation of ceramide. Pretreatment with sphingomyelinase inhibitors blocked ceramide generation, decreases in phospho-Akt, nanovesicle release and cell detachment in response to alkylphospholipids and PIAs in non-small cell lung cancer cell lines. Similarly, exogenous ceramide also decreased active Akt and induced nanovesicle release. Knockdown of neutral sphingomyelinase decreased, whereas overexpression of neutral or acid sphingomyelinase increased cell detachment and death in response to the compounds. When transferred in vitro, PIA or Per-induced nanovesicles increased ceramide levels and death in recipient cells. These results indicate ceramide generation underlies the Akt inhibition and cytotoxicity of this group of agents, and suggests nanovesicle shedding and uptake might potentially propagate their cytotoxicity in vivo.

Cell Death and Disease (2012) 3, e340; doi:10.1038/cddis.2012.72; published online 5 July 2012

Subject Category: Cancer

Akt has a central role in the phosphoinositide-3-kinase (PI3K)/ Akt signaling pathway that controls cellular processes integral in the development of cancer including growth, metabolism and survival. As Akt promotes therapeutic resistance and is active in a majority of human cancers, inhibitors are being developed as cancer therapeutics. Akt is activated in response to the lipid products of class I $\mathrm{PI} 3 \mathrm{~K}, \mathrm{PI}, 4 \mathrm{P}_{2}$ (PIP2) and $\mathrm{PI}, 4,5 \mathrm{P}_{3}$ (PIP3). Once synthesized, PIP2 and PIP3 attract Akt to the plasma membrane through binding to the pleckstrin homology $(\mathrm{PH})$ domain of Akt. This promotes a conformational change, allowing it to be phosphorylated on T308 by PDK-1 and at $\mathrm{S} 473$ by the rictor/mTOR complex. Once phosphorylated, Akt dissociates from the plasma membrane and moves to various cellular compartments where it phosphorylates downstream substrates.

Most Akt inhibitors in development target the ATP-binding region, whereas lipid-based inhibitors such as phosphatidylinositol ether lipid analogs (PIAs) and the alkylphospholipids perifosine (Per) and miltefosine were designed to interfere with the $\mathrm{PH}$ domain of Akt. In addition to decreasing active Akt, PIAs have pleiotrophic effects in cancer cells including p38 and AMPK activation, p21 and RhoB induction, ${ }^{1-4}$ cellular detachment and apoptosis. In vitro, PIAs are broadly cytotoxic and share a similar cytotoxicity pattern with alkylphospholipids. ${ }^{5}$ Per also inhibits Akt, and is currently being evaluated in phase III clinical trials. In addition, alkylphospholipids can activate JNK, target cell membranes, induce p21 in a p53-independent manner and disturb lipid rafts. ${ }^{6}$ Single-agent activity with Per has been observed in sarcoma and Waldenstrom Macroglobinulemia (WM) patients. However, the poor response rates of a majority of common solid tumors to Per as a single agent has lowered expectations and prompted further investigation into its mechanism of action. ${ }^{6}$

In this study, we show both types of compounds stimulate release of nanovesicles containing active Akt, EGFR and IGF-IR and the tetraspanins CD151 and CD81 from tumor cells in vitro and in vivo through sphingomyelinase-mediated generation of ceramide. PIA and Per induced decreases in P-Akt, cell detachment and death also depended on ceramide generation. Our data suggests ceramide is a crucial mechanism that ties together many of known activities of these agents. As ceramide induction is required for cytotoxicity, the status of intratumoral sphingolipid metabolism may determine clinical response.

\footnotetext{
${ }^{1}$ Medical Oncology Branch, Center for Cancer Research, National Cancer Institute, Bethesda, MD 20892, USA; 'Laboratory of Pathology, Center for Cancer Research, National Cancer Institute, Bethesda, MD 20892, USA; ${ }^{3}$ Department of Pulmonary Medicine, University of California at Davis School of Medicine, Sacramento, CA 95817, USA and ${ }^{4}$ Department of Medicinal Chemistry and Pharmacognosy, College of Pharmacy, University of Illinois at Chicago, Chicago, IL 60612, USA

${ }^{*}$ Corresponding author: PA Dennis, Medical Oncology Branch, Center for Cancer Research, National Cancer Institute, 37 Convent Drive, Room 1042, Bethesda, MD 20892-4254, USA. Tel: +301 496 0929; Fax: +301 435 4345; E-mail: pdennis@ @ih.gov

Keywords: Akt; nanovesicle; sphingomyelinase; ceramide; perifosine

Abbreviations: NSCLC, non-small cell lung cancer; P5, PIA5; Per, perifosine; SMPD1, acid sphingomyelinase; SMPD3, neutral sphingomyelinase; LY, LY294002; MCD, methylcyclodextrin

Received 10.10.11; revised 13.4.12; accepted 2.5.12; Edited by M Piacentini
} 


\section{Results}

PIAs inhibit EGF- and IGF-I-stimulated pathway activation and decrease expression of EGFR and IGF-IR. Our initial aim was to investigate whether PIAs could inhibit growth factor induced, as well as endogenous Akt activation in cancer cells. To assess this, $\mathrm{H} 157$ cells were pre-treated with PIA5 (P5) then stimulated with EGF and harvested for immunoblotting (Figure 1a). EGF increased p-EGFR and p-Akt S473, but decreased the amount of total EGFR. Pretreatment with P5 diminished the EGF-induced increase in p-Akt at S473 and T308, and also unexpectedly decreased the phosphorylation of EGFR. P5 alone decreased total EGFR levels to a similar extent as EGF treatment, while the combination of PIA plus EGF caused the greatest decrease in total EGFR. Similar results were obtained with IGF-I stimulation (Figure 1b). P5 pretreatment inhibited IGF-I-stimulated p-Akt, p-IGFR, and decreased the total level of IGF-IR without affecting total Akt. These data suggest PIAs have effects on membrane proteins proximal to the PI3K/Akt pathway, and that PIA-induced Akt inhibition may be due in part to depletion of growth factor receptor activation that is upstream of Akt.

To confirm that PIAs decrease expression of EGFR and IGF-IR, time course experiments were performed in three non-small cell lung cancer (NSCLC) cell lines (Figure 1c). In each cell line, P5 caused a rapid and progressive decrease in EGFR and IGF-IR. This correlated with a decrease in p-Akt (S473) but not total Akt. PIAs also caused an early and sustained phosphorylation of p38, which is a known activity of PIAs that is independent of Akt inhibition. ${ }^{2}$ This suggests that loss of EGFR and IGFR expression by PIAs is a common cellular response.

PIAs, Per and a cholesterol-depleting agent, but not a PI3K inhibitor, decrease growth factor receptor expression and activate p38. To assess whether decreases in EGFR and IGF-IR expression were common to all known active PIAs or to other inhibitors of the PI3K/Akt pathway, P5,6,23,24 and 25 were compared against Per or a PI3K inhibitor (LY294002 (LY)). Methylcyclodextrin (MCD), a cholesterol chelator that is commonly used to disrupt lipid rafts and decreases p-Akt, ${ }^{7}$ was also included. By $1 \mathrm{~h}$, all active PIAs and Per decreased total EGFR and IGF-IR levels, inhibited Akt phosphorylation and increased p38 phosphorylation (Figure 2). In contrast, LY decreased Akt phosphorylation but did not affect EGFR, IGF-IR or p-p38 at any of the time points examined. MCD had similar effects as PIAs, but with different kinetics. MCD decreased $p$-Akt by $1 \mathrm{~h}$, but only caused activation of p38 and decreased expression of EGFR and IGF-IR at $4 \mathrm{~h}$. These data indicate that loss of EGFR and IGF-IR is a common feature of lipidbased Akt inhibitors that is not shared with other pathway inhibitors, and that PIAs and Per inhibit Akt in a PI3Kindependent manner. The similarity of effects between PIAs, Per and MCD suggests that some of the effects of the lipidbased Akt inhibitors could be through disruption of lipid rafts or membrane structure.
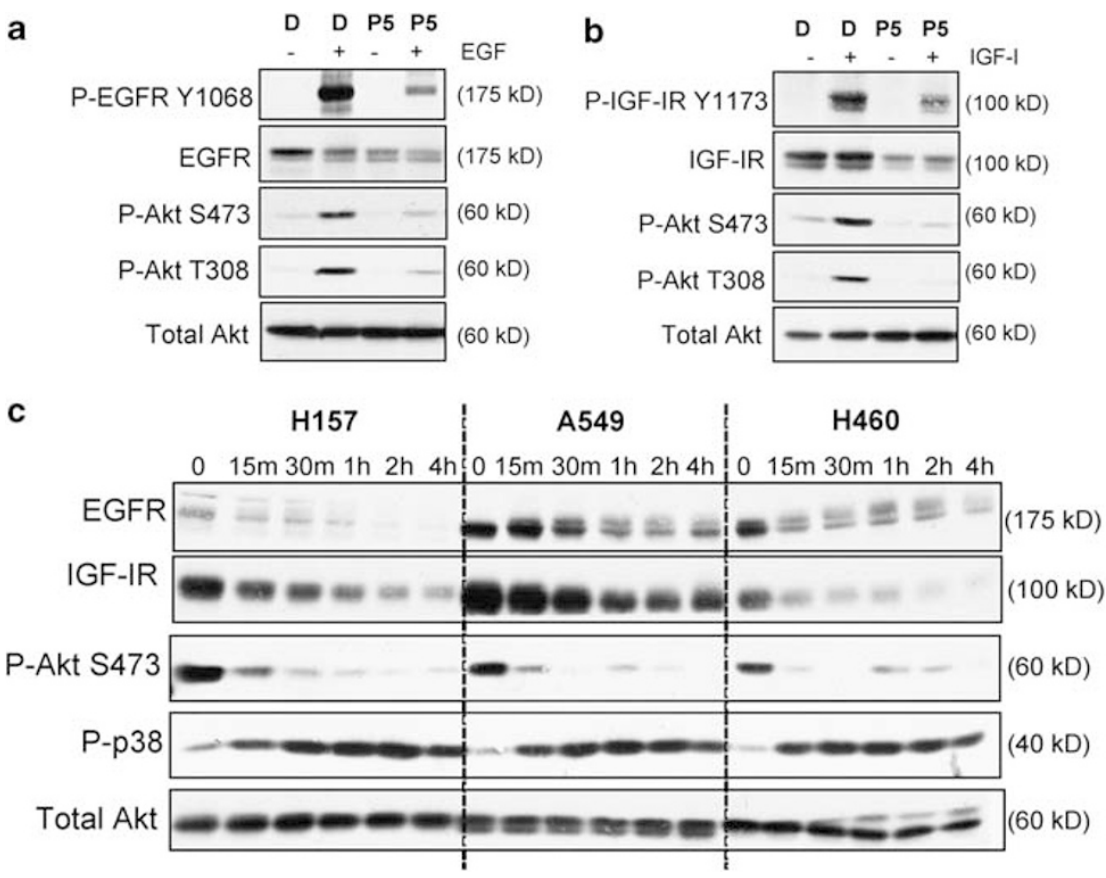

Figure 1 P5 blocks growth factor stimulation of P-Akt and decreases the expression of growth factor receptors in NSCLC cells. (a) P5 inhibits EGF-stimulated P-EGFR, P-Akt and decreases total EGFR levels. H157 cells were pre-treated with $10 \mu \mathrm{M}$ P5 or DMSO (D) for $30 \mathrm{~min}$ followed by stimulation with $100 \mathrm{ng} / \mathrm{ml}$ EGF for $15 \mathrm{~min}$. Immunoblotting of cell lysates was performed as in the Materials and Methods section. (b) P5 inhibits IGF-I-stimulated Akt and IGF-IR phosphorylation and decreases total IGF-IR expression. H157 cells were pre-treated with P5 as in A, followed by stimulation with $50 \mathrm{nM} \mathrm{IGF-I} \mathrm{for} 15$ min, followed by immunoblotting. (c) P5 treatment increases P-p38 and causes rapid decreases in total EGFR, IGF-IR and P-Akt, but does not decrease total Akt levels. H157, A549 and H460 NSCLC cells were treated with $10 \mu \mathrm{M}$ P5 for the indicated times and analyzed by immunoblot 

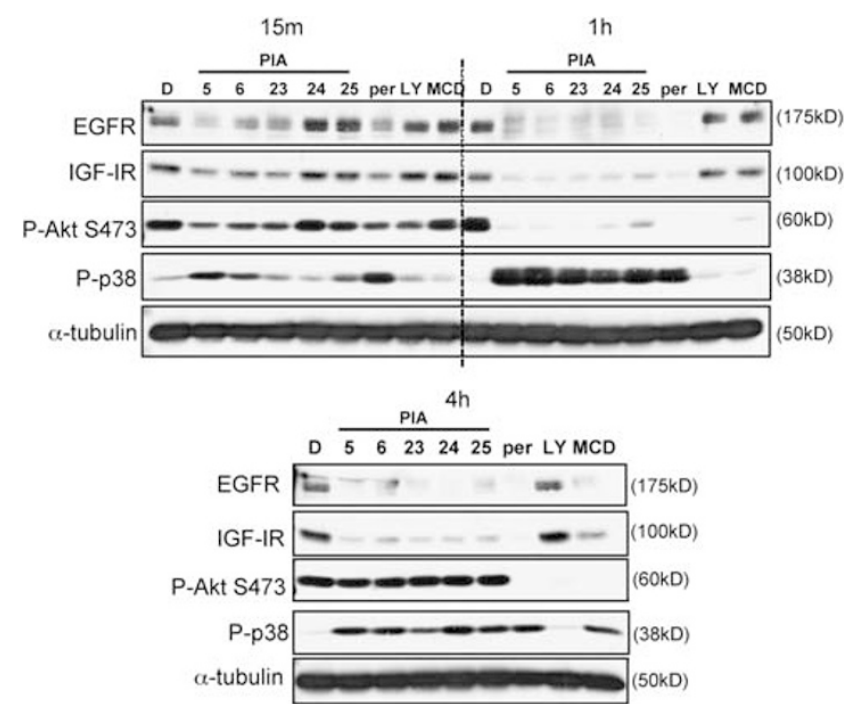

Figure 2 PIAs, Per and MCD, but not LY, decrease growth factor receptors and activate p38. Immunoblot of $\mathrm{H} 157$ cells treated with DMSO (D), $10 \mu \mathrm{M}$ PIA $5,6,23,24$ or $25(5,6,23,24,25), 10 \mu \mathrm{M}$ Per, $10 \mu \mathrm{M}$ LY or $10 \mathrm{mM} \mathrm{MCD}$ for the indicated times

Full-length EGFR, IGF-IR and P-Akt are present in the media of PIA and Per-treated, but not vehicle-treated cells. The loss of EGFR and IGFR expression suggested these proteins were possibly being degraded. However, pretreatment with a proteasome inhibitor (Supplementary Figure S2A) or a lysosome inhibitor (Supplementary Figure S2B) failed to block the loss of EGFR or IGFR by PIAs. Therefore, secretion of these proteins into the culture media was assessed. Media from cells treated with DMSO, P5, Per or MCD for $1 \mathrm{~h}$ were collected and concentrated, then immunoblotting of a portion of the media and cell lysates was performed (Figure 3a). In A549 and $\mathrm{H} 157$ cells, P5 or Per decreased levels of EGFR and IGF-IR in the cell lysates, and increased their levels in the media. As the electrophoretic mobility of the proteins did not change, which would imply proteolysis, we hypothesized these proteins might be co-localized in shed vesicles. Differential centrifugation was used to separate media contents. Media components were separated by a series of low-speed spins to remove floating cells and debris, followed by ultracentrifugation at $100000 \times g$ for $1 \mathrm{~h}$. The remaining $100000 \times g$ supernatants were concentrated and separated via SDS-PAGE electrophoresis, along with the $100000 \times g$ media pellet and the cell lysate (Figure 3b). Following centrifugation, EGFR, IGFR and p-Akt, but not p-p38 were concentrated in the $100000 \times g$ pellet from PIA and Per, but not vehicle or LY-treated cells, suggesting that PIAs and Per caused EGFR, IGF-IR and $P$-Akt release in a vesicle.

To assess the location of subcellular contents after PIA or Per treatment, an equal quantity of protein from each of the media pellets were loaded on a SDS-PAGE gel for immunoblotting (Figure 3c). Markers of the early endosome (EEA1), lysosome (lamp2), endoplasmic reticulum (bip), nucleus (lamin A/C) and mitochondria (cox IV) were present in the cell lysate and the $300 \times g$ pellet (which represents the floating cells), but were absent from the $10000 \times g$ and $100000 \times g$ pellets. The 10000 and $100000 \times g$ pellets were highly enriched in CD151 and CD81, tetraspanins that are indicators of nanovesicles derived from an endosomal origin, ${ }^{8}$ as well as a marker of lipid rafts, Gi $22 .{ }^{9}$ Treatment of $\mathrm{H} 460$ and A549 cells with P5 or Per caused a similar release of EGFR, IGF-IR, Gi 22, CD151, p-Akt and CD81 that was captured primarily in the $100000 \times g$ pellets (Supplementary Figure S3).

PIA and Per-induced nanovesicle release does not depend on active Akt. Since Akt has a role in GLUT vesicle trafficking, the role of Akt in PIA and Per-induced vesicle release was assessed. $\mathrm{H} 157$ cells were pre-treated with LY, followed by P5 or Per treatment for $1 \mathrm{~h}$ (Supplementary Figure S4). Although LY decreased p-Akt in the cell lysate, it did not alter the ability of P5 or Per to increase levels of EGFR, IGFR, total Akt, CD151 or CD81 in the media, indicating that active Akt is not required for vesicle release induced by these compounds.

Rapid visualization of nanovesicles released by lipidbased Akt inhibitors. To visualize the release of vesicles in real-time, live $\mathrm{H} 157$ cells were stained with FM 1-43X and imaged by time-lapse confocal microscopy. Addition of P5 increased release of fluorescent vesicles into the media within $15 \mathrm{~s}$ (Supplemental videos S1 and S2). To determine the morphology of these nanovesicles, transmission electron microscopy of the $100000 \times g$ media pellet from $1 \mathrm{~h}$ P5- and Per-treated $\mathrm{H} 157$ cells was performed (Supplementary Figure S5). The micrographs revealed that the pellets contained heterogeneous vesicles whose diameters ranged between $50 \mathrm{nM}$ and $1 \mu \mathrm{M}$. These results show these compounds rapidly induce the release of nanovesicles from cancer cells.

Role of ceramide. Ceramide has been implicated in multivesicular endosome production and release of exosomes. ${ }^{10}$ To assess whether P5 might increase nanovesicle release through ceramide generation, cellular ceramide was measured using a diacylglycerol kinase assay (Supplementary Figure S7). P5 increased ceramide levels by twofold. PIA7, an inactive analog, ${ }^{1}$ did not increase ceramide levels. Ceramide was also assessed via immunofluorescent staining using an anti-ceramide antibody (Figure 4a). Treatment of $\mathrm{H} 157$ cells with PIA23, Per and miltefosine for $6 \mathrm{~h}$ markedly induced ceramide in the cells, as indicated by the increased red color. This induction was blocked by pretreatment with a neutral sphingomyelinase (SMPD3) inhibitor (GW4869), or acid sphingomyelinase (SMPD1) inhibitor (desipramine), suggesting that ceramide induction is through activation of sphingomyelinases and not de novo synthesis. To confirm that sphingomyelinases were being activated, sphingomyelinase activity was measured. Treatment of $\mathrm{H} 157$ cells with P5 and Per for $1 \mathrm{~h}$ significantly increased sphingomyelinase activity at $\mathrm{pH} \mathrm{7,} \mathrm{and} \mathrm{this} \mathrm{was} \mathrm{blocked} \mathrm{by} \mathrm{GW4869} \mathrm{pretreatment}$ (Figure 4b). In contrast, none of the compounds significantly increased sphingomyelinase activity at $\mathrm{pH} 5$ after $1 \mathrm{~h}$, however, there was a trend toward an increase with both Per and miltefosine that could be blocked by desipramine pretreatment (Figure 4c). As GW4869 and desipramine are reported to inhibit different isoforms of sphingomyelinase, 

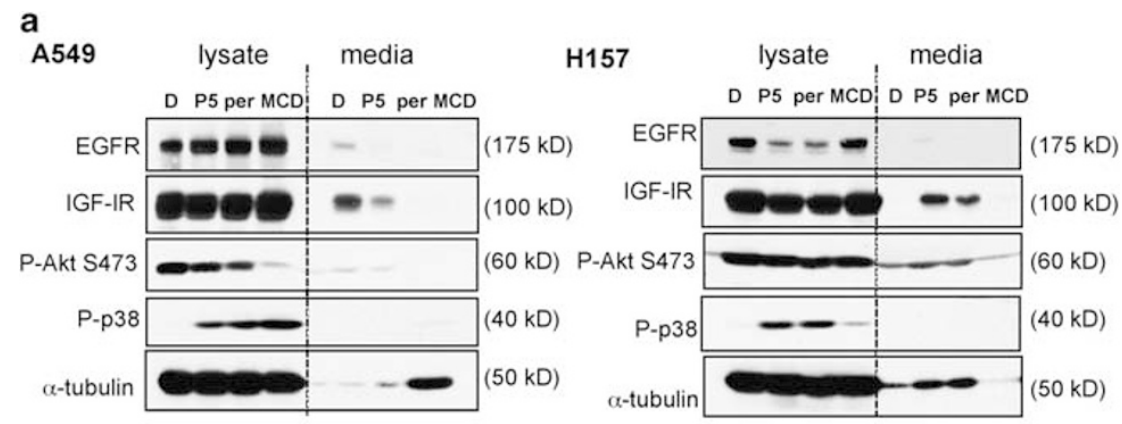

b

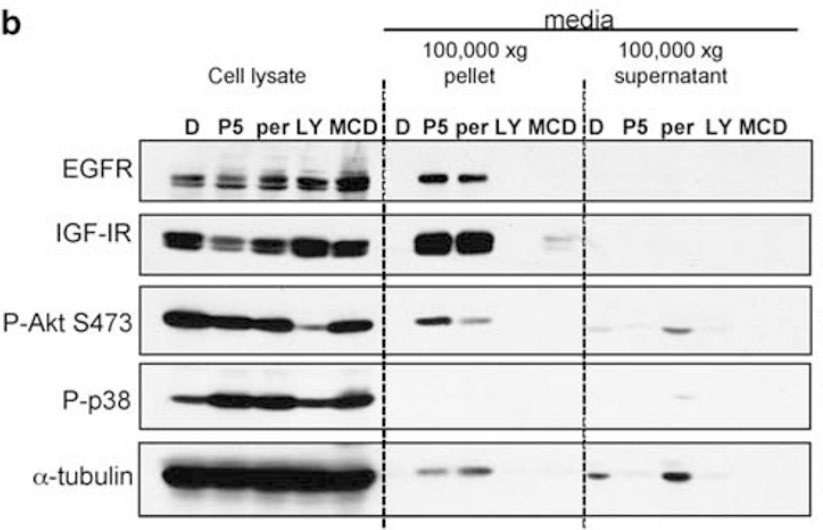

(175 kD)

C

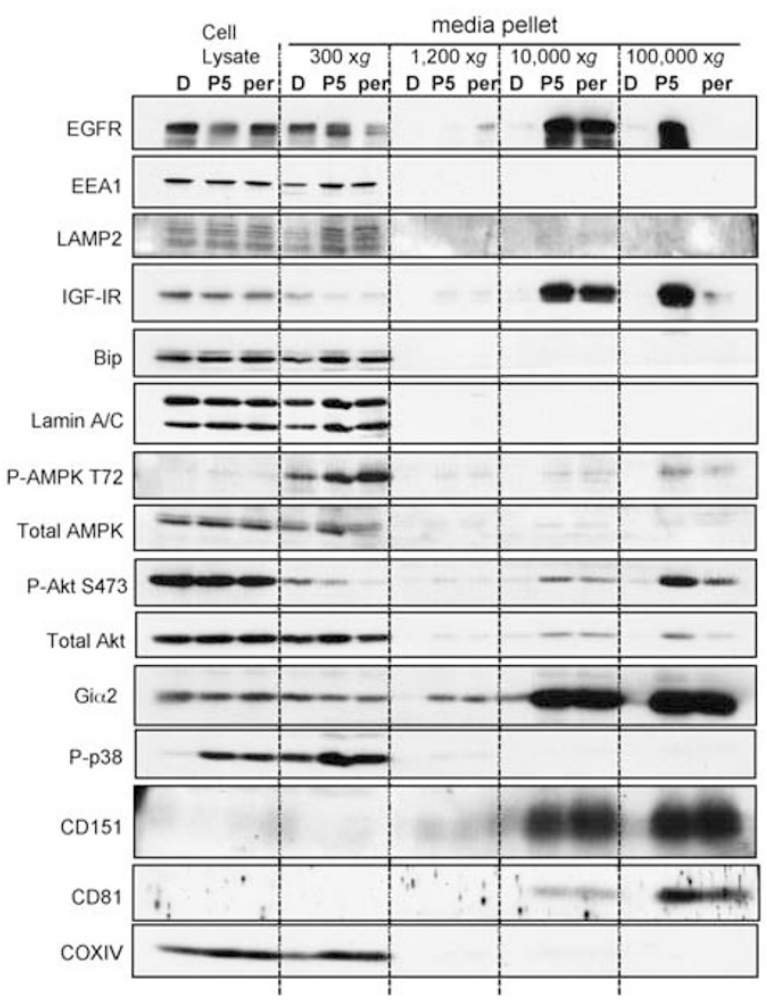

Figure 3 (a) EGFR, IGF-IR and P-Akt are present in the extracellular media following P5 or Per treatment. A549 and H157 cells were treated with DMSO (D), P5, Per or MCD for $1 \mathrm{~h}$; cell culture media were concentrated using a Centricon Ultracel YM-10 filter unit (Millipore), and an equal amount of protein from the cell lysate and media were analyzed by immunoblot. (b) EGFR, IGF-IR and P-Akt are present in the $100000 \times g$ pellet from PIA- or Per-treated cell conditioned media. H157 cells were treated as in A, media were collected and centrifuged at $300 \times g(10 \mathrm{~min}), 1200 \times g(20 \mathrm{~min}), 10000 \times g(30 \mathrm{~min})$ and $100000 \times g(1 \mathrm{~h})$ then equal protein from the cell lysate, the $100000 \times g$ media pellet and the $100000 \times g$ supernatant were analyzed by immunoblot. (c) The $100000 \times g$ media pellets from PIA or Per-conditioned media are enriched in the tetraspanins CD81 and CD151, and the raft marker Gi 2 2, but do not contain markers of the early endosome (EEA1), lysosome (lamp2), nucleus (lamin A/C), endoplasmic reticulum (bip) or mitochondria (COXIV). H157 cells were treated with DMSO (D), P5 or Per for $1 \mathrm{~h}$. The media were collected and centrifuged as in (b), followed by immunoblot analysis of equal amounts of protein from the cell lysate and media pellets 


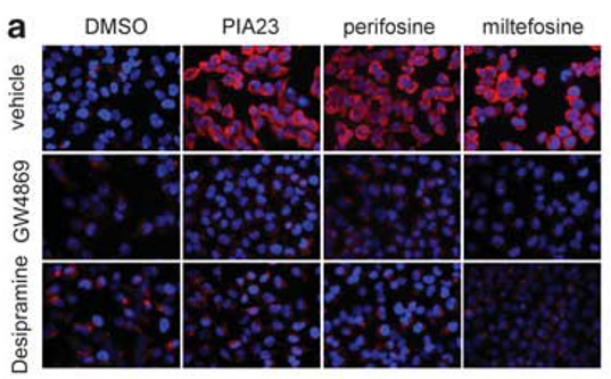

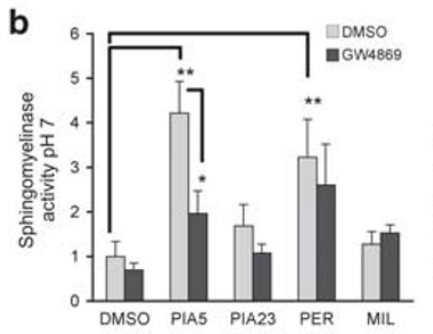

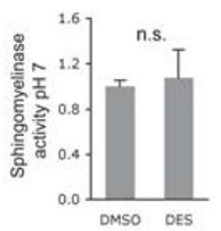

C
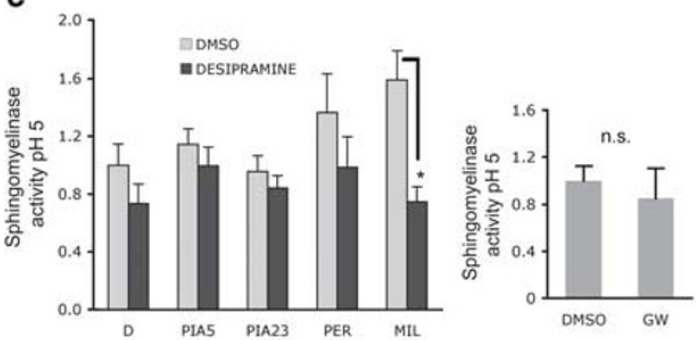

d
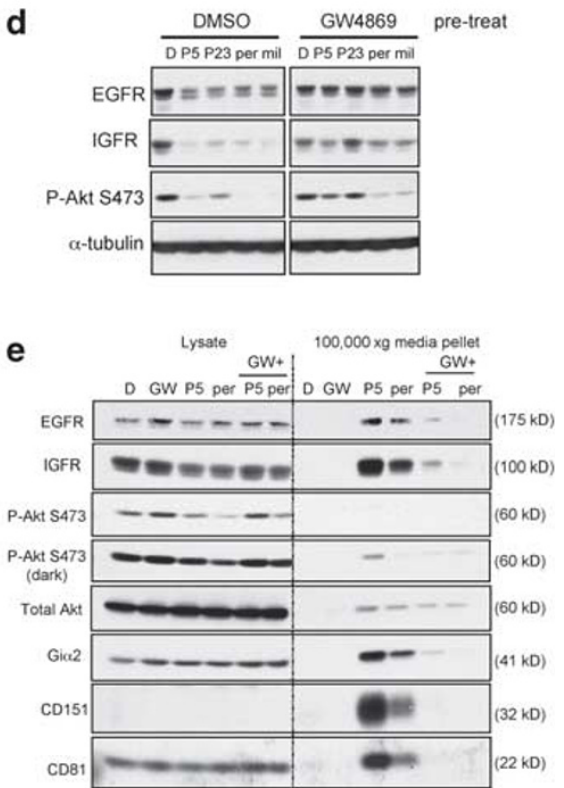

f

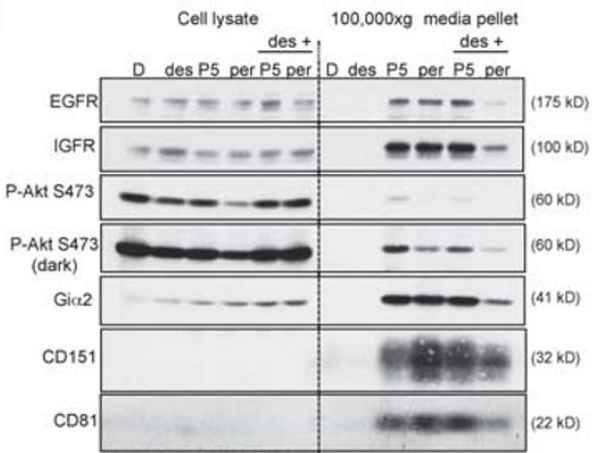

g

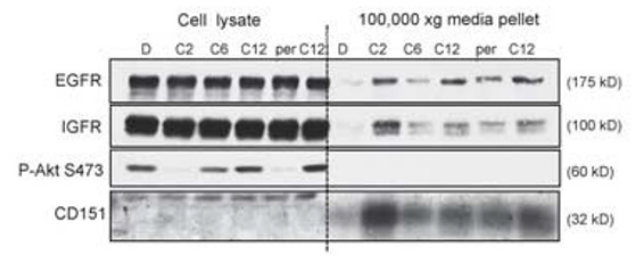

Figure 4 (a) PIA23, Per and miltefosine treatments $(6 \mathrm{~h})$ increase ceramide that may be blocked by sphingomyelinase inhibitors. Shown are fluorescent micrographs of $\mathrm{H} 157$ cells stained with an anti-ceramide antibody (red), nuclei are blue (DAPI). (b) Incubation with PIAs or Per increases SMPD3 activity. Cells were pre-treated with DMSO or $5 \mu \mathrm{M}$ GW4869 for $2 \mathrm{~h}$, followed by treatment with PIAs or alkylphospholipids for $1 \mathrm{~h}$. Spingomyelinase activity in cell lysates was measured at $\mathrm{pH} 7,{ }^{\star} P<0.5,{ }^{* *} P<0.01$. (c) Incubation with alkylphospholipids increases SMPD1 activity. Cells were pre-treated with DMSO or $25 \mu \mathrm{M}$ desipramine for $2 \mathrm{~h}$ followed by treatment with PIAs and alkylphospholipids for $1 \mathrm{~h}$. A two-step sphingomyelinase activity was performed at $\mathrm{pH} 5,{ }^{*} P<0.05$. (d) GW4869 pretreatment decreases PIA or alkylphospholipid induced decrease in growth factor receptors and phospho-Akt in whole cell lysates. (e) PIA and Per-induced vesicle release is blocked by an inhibitor of SMPD3. H157 cells were pre-treated with $2.5 \mu \mathrm{M}$ GW4869 for $30 \mathrm{~min}$, followed by 1-h treatment with DMSO, P5 or Per. Media were collected and centrifuged as described in the Materials and Methods section. (f) Pretreatment with desipramine, an SMPD1 inhibitor, decreases release of nanovesicles induced by Per. (g) Treatment with exogenous ceramide leads to nanovesicle release. $\mathrm{H} 157$ cells were treated with DMSO (D), $25 \mu \mathrm{M} \mathrm{C2}, \mathrm{C} 6$ or $\mathrm{C} 12$ ceramide or $5 \mu \mathrm{M}$ Per for $8 \mathrm{~h}$. Media were centrifuged as described in the Materials and Methods section. Des $=50 \mu \mathrm{M}$ desipramine; P5 $=10 \mu \mathrm{M}$ P5; and Per $=10 \mu \mathrm{M}$ Per

we confirmed that desipramine alone did not significantly decrease sphingomyelinase activity at $\mathrm{pH} 7$ (Figure 4b, right panel), nor did GW4869 alter sphingomyelinase activity at $\mathrm{pH}$ 5 (Figure 4c, right panel).

To assess if blocking ceramide could alter the loss of growth factor receptors or P-Akt, cells were pre-treated with
GW4869, followed by treatment with P5, PIA23, Per or miltefosine for $1 \mathrm{~h}$ (Figure 4d). Pretreatment with GW4869 blocked the loss of EGFR, IGFR and P-Akt from cells, suggesting that ceramide was leading to nanovesicle release. To confirm this, the experiment was repeated and contents of the media were assessed (Figure 4e). GW4869 pretreatment 
blocked PIA and Per induced decreases in P-Akt in the cell lysates, and inhibited the increase in EGFR, IGFR, Gi 2 , CD151 and CD81 released into the media, strongly suggesting that ceramide generation by SMPD3 is driving the nanovesicle release. Similar results were obtained with the A549 cell line (Supplementary Figure S8). Pretreatment of H157 cells with an SMPD1 inhibitor blocked the cellular decrease in P-Akt, and partially abrogated the nanovesicle release in response to Per, but not P5 (Figure 4f). These differences could be explained by differences in specificity of the inhibitors and/or differences in affinity for classes of sphingomyelinases.

To assess whether exogenous ceramide could recapitulate effects of alkylphospholipids and PIAs, $\mathrm{H} 157$ cells were treated with $\mathrm{C} 2, \mathrm{C} 6$ or $\mathrm{C} 12$ ceramide, and the contents of the $100000 \times g$ pellet were analyzed. All three forms of ceramide increased levels of EGFR and IGF-IR in nanovesicles, but the greatest increases were observed with $\mathrm{C} 2$ ceramide. Levels of EGFR and IGF-IR in nanovesicles induced by exogenous ceramide were similar to those induced by Per (Figure $4 \mathrm{~g}$ ). These results indicate that exogenous ceramide can cause nanovesicle release, and are consistent with the observation that PIAs and alkylphospholipids cause nanovesicle release by stimulating sphingomyelinase activity and generating ceramide.

Ceramide leads to cell detachment and death. As ceramide causes morphological changes ${ }^{11}$ and death, we treated cells with the SMPD3 inhibitor, followed by PIA23, Per or miltefosine. After $6 \mathrm{~h}$, cells began to round up and detach, and this was blocked by pretreatment with GW4869 (Figure 5a). GW4869 also inhibited morphological changes induced by P5 (Figure 5a, lower images), but not exogenous C2 ceramide, which still caused cell detachment and death even in the presence of the SMPD3 inhibitor. To confirm that SMPD3 is involved in the mechanism of ceramide generation and death, siRNA against SMPD3 was transfected into $\mathrm{H} 157$ cells. Knockdown of SMPD3 greatly abrogated the morphological changes (Figure 5b, top) and inhibited death (Figure 5b, bottom left panel) in response to PIAs, Per or miltefosine. Whereas knockdown of SMPD1 only protected against Per and miltefosine-induced death (Figure $5 \mathrm{c}$, bottom panel), and did not cause changes in cell morphology with treatment (Figure 5c, top panels). Next, we overexpressed V5-tagged neutral and SMPD1 in H157 cells and assessed the impact on PIA- or Per-induced cell death (Figure $5 \mathrm{~d}$ ). Overexpression of either isoform increased cell rounding and cell detachment induced by the compounds. SMPD3 increased death in response to PIA23 and miletosine, but only increased death in response to Per slightly. In this experiment, P5 caused complete cell death, therefore the impact of SMPD3 or SMPD1 overexpression could not be assessed. SMPD1 transfection greatly increased death in response to PIA23, Per and miltefosine. As SMPD1 overexpression increased and SMPD1 knockdown decreased death in response to Per, this may suggest that acid sphingomyelinse is the more important isoform responsible for ceramide generation and Per-induced cell death.

P5 and Per increase circulating levels of tumor-derived nanovesicles. As PIAs and Per are being developed as cancer therapeutics, we assessed the release of nanovesicles in an in vivo tumor model. Athymic mice bearing H157 tumor xenografts were given a single intraperitoneal (i.p.) injection of $90 \mathrm{mg} / \mathrm{kg}$ P5 or vehicle, blood plasma was harvested $1 \mathrm{~h}$ later and nanovesicles were isolated using differential centrifugation. Similar to our in vitro results, P5 greatly increased levels of EGFR, IGFR and P-Akt S473 in the $100000 \times g$ plasma pellet of P5-treated mice (Figure 6a and Supplementary Figure S6A), confirming that nanovesicle release also occurs in vivo. P5 also decreased p-Akt levels in the tumors of the mice (Supplementary Figure S6A, bottom panel). To confirm that nanovesicles were being shed from tumor cells, $\mathrm{H} 157$ cells were stably transfected with an EGFR-GFP fusion protein, and transfected cells were injected subcutaneously into nude mice and grown as xenografts. Mice were treated i.p. with vehicle, $90 \mathrm{mg} / \mathrm{kg}$ P5 or $40 \mathrm{mg} / \mathrm{kg}$ Per. Plasma was harvested $1 \mathrm{~h}$ later, and analysis of the $100000 \times g$ plasma pellets was performed as before. P5 and Per increased tumor-derived, GFP-tagged EGFR in the $100000 \times g$ pellet, suggesting that vesicles were shed from the tumors (Supplementary Figure S6B).

PIA or Per-induced nanovesicles can be taken up by recipient cells. Because the fate of circulating nanovesicles is unknown and because recent studies suggest cellular nanovesicles have a role in intercellular communication, we assessed whether nanovesicles shed in response to PIA or Per could be taken up by recipient tumor cells. $\mathrm{H} 157$ cells stably transfected with EGFR-GFP were treated with DMSO, P5 or Per for $1 \mathrm{~h}$. The media from these cells were centrifuged at the different speeds, and the $100000 \times g$ media pellets were added to recipient $\mathrm{H} 157$ cells that did not express EGFR-GFP. Nanovesicle uptake was monitored using fluorescence microscopy. Within a few hours, cells that received the nanovesicle fraction from PIA or Per-treated donor cells exhibited increased intracellular fluorescence. Cells that received the pellet from DMSO-treated donor cells did not show increased fluorescence. Fluorescent labeling of the recipient cells persisted after the cells were extensively washed and refed with media, suggesting the cells had incorporated the nanovesicle contents (Figure 6b).

PIA or Per-induced nanovesicles decrease cell proliferation and induce ceramide when transferred to recipient cells. The fact that P5 or Per-induced nanovesicles contain proteins such as EGFR, IGFR and p-Akt suggests that uptake of nanovesicles by recipient cells might promote cell growth or survival. To test this, the $100000 \times g$ media pellet from 1-h-treated EGFR-GFP H157 donor cells was collected, washed with PBS and given to recipient $\mathrm{H} 157$ cells for $72 \mathrm{~h}$, at which time proliferation was assessed. Nanovesicles were collected from either one or three donor flasks. Nanovesicles from PIA- or Per-treated cells decreased proliferation of recipient cells in a dose-dependent manner (Figure 6c). Recipient cells also became refractile and looked unhealthy with longer incubation times. Increased refractivity is also observed when PIAs or Per are incubated with cells directly, suggesting that there might be carryover of the compounds within nanovesicles that is transferred to the recipient cells. 
a
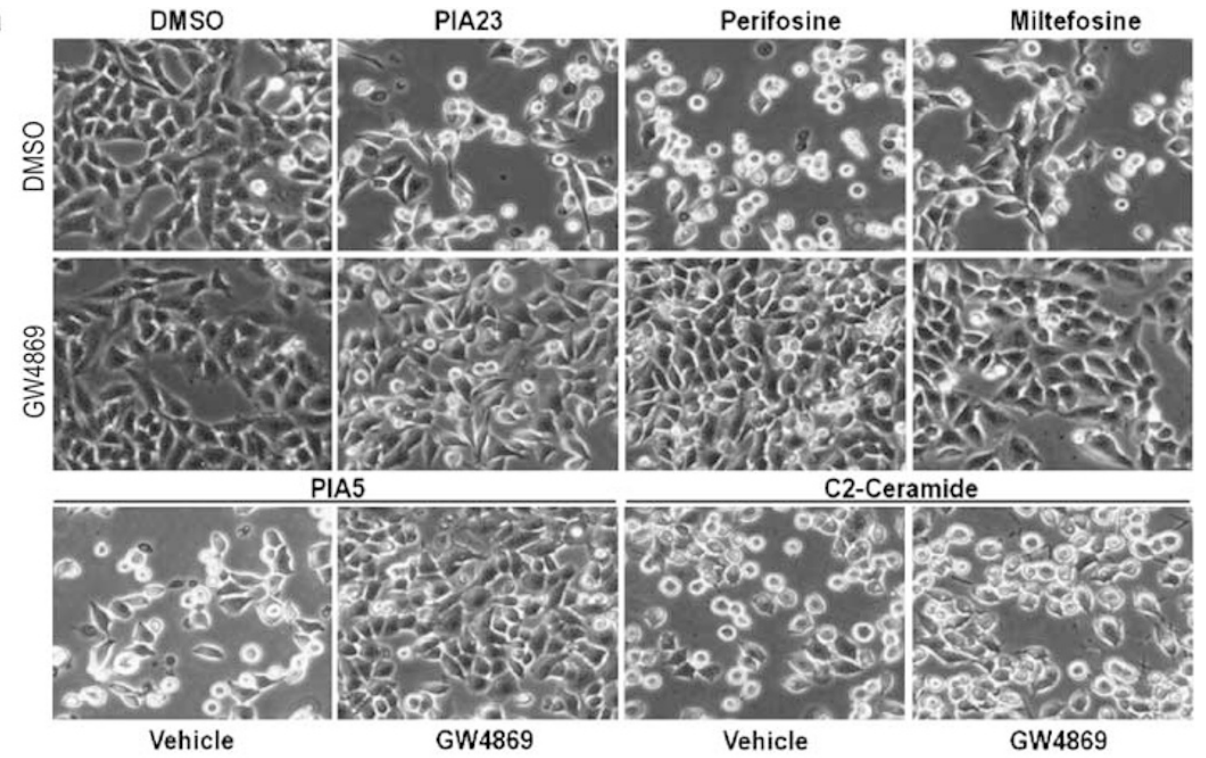

b

b DMSO

PIA23

Perifosine

Milfefosine

PIA5
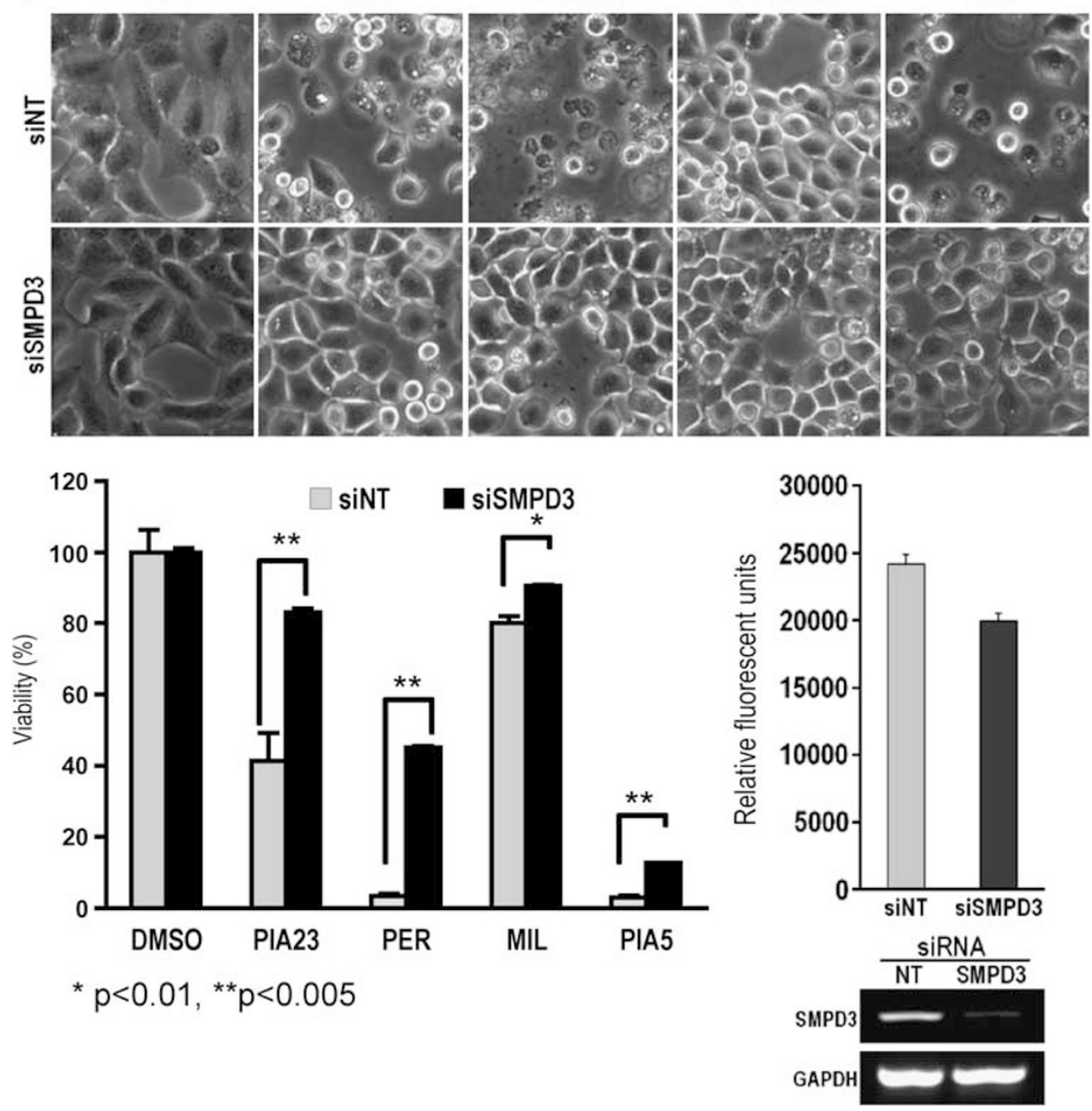

Figure 5 Continued 

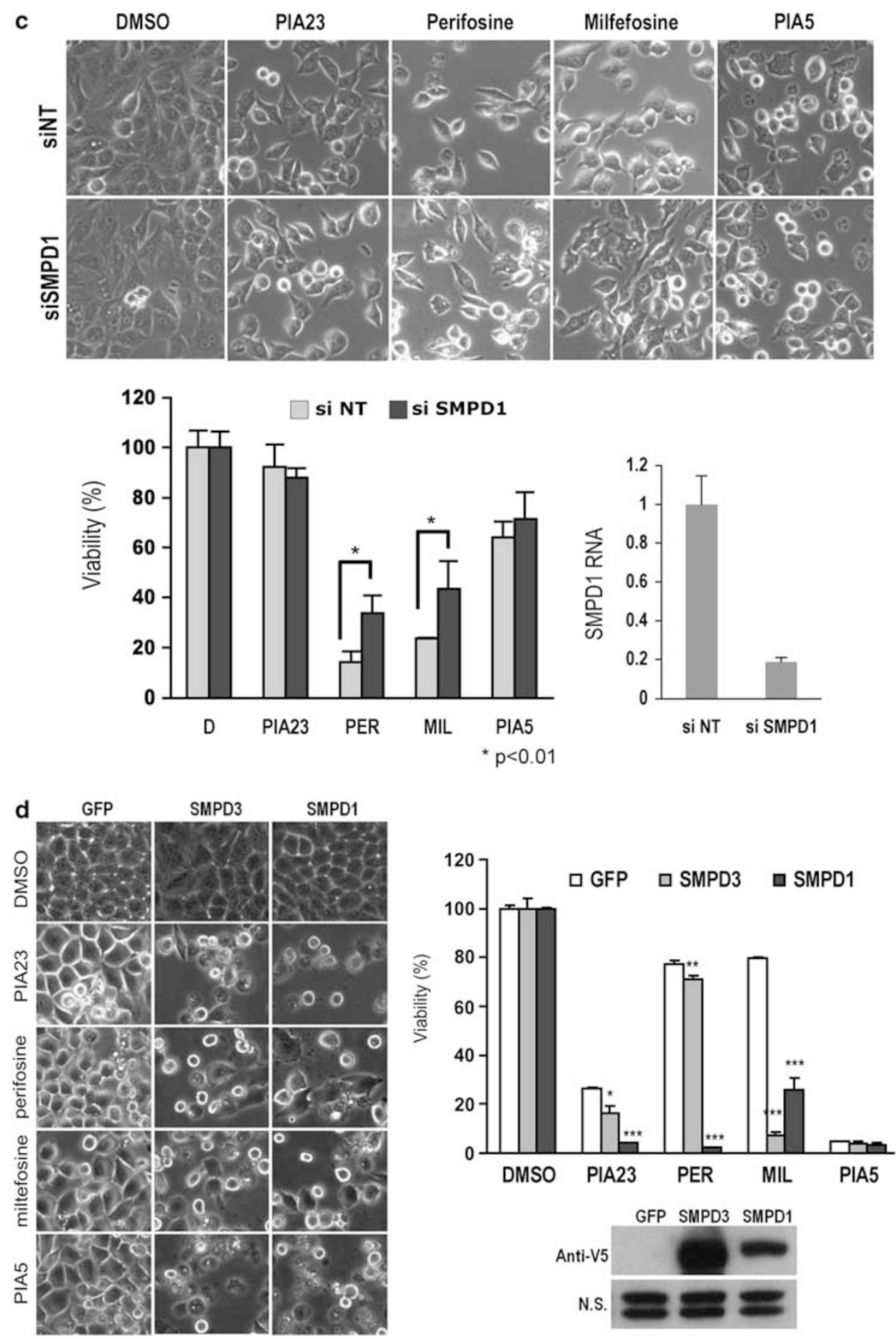

Figure 5 (a) Treatment with P5, PIA23, Per or miltefosine causes cell detachment that is blocked by a SMPD3 inhibitor (top panels), in contrast to C2 ceramide. (b) Knockdown of SMPD3 protects $\mathrm{H} 157$ cells against death induced by PIAs or alkylphospholipids. siNT, nontargeting siRNA; siSMPD3, siRNA against SMPD3. Controls for the knockdown are shown on the right. Top right panel is an assay for SMPD3 activity following siRNA transfection. Lower right panel depicts RT-PCR for SMPD3 mRNA after siRNA transfections. (c) Knockdown of SMPD1 protects against death induced by alkylphospholipids. siNT, nontargeting siRNA; siSMPD1, siRNA against SMPD1. (d) Overexpression of SMPD3 or SMPD1 potentiates the death caused by incubation with PIAs, Per and miltefosine. Cells were transiently transfected with V5-tagged plasmids to SMPD3 or SMPD1. ${ }^{\star} P<0.01,{ }^{\star \star} P<0.001$ and ${ }^{* \star \star} P<0.0001$

To assess this, recipient cells were pre-treated or not with the SMPD3 inhibitor GW4869 for $2 \mathrm{~h}$, followed by the $100000 \times g$ media pellet from P5- or Per-treated EGFRGFP H157 cells. Ceramide generation was assessed with an anti-ceramide antibody. Staining with an anti-ceramide antibody showed increased ceramide in the recipient cells that could be blocked by GW4869 pretreatment, suggesting the presence of the compounds in the donor pellet (Figure 6d). 

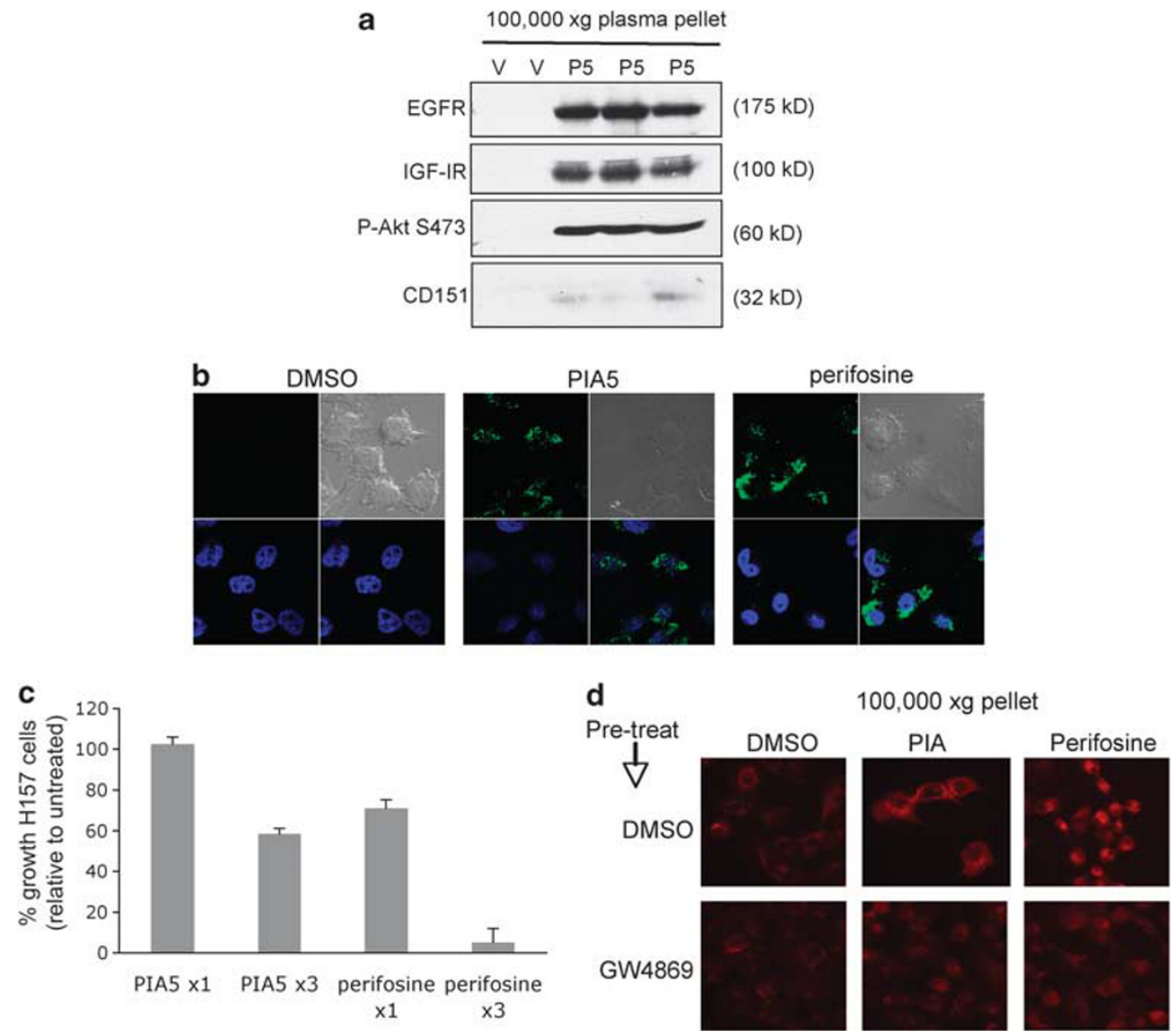

Figure 6 PIA and Per cause nanovesicle release in vivo. (a) Athymic mice bearing $\mathrm{H} 157$ xenografts were given a single i.p. injection of vehicle or $90 \mathrm{mg} / \mathrm{kg} \mathrm{P5}$. After $1 \mathrm{~h}$, cardiac puncture was performed; plasma was isolated and subjected to differential centrifugation as in the Materials and methods section. Shown are the $100000 \times g$ plasma pellets from two vehicle (V), or three P5-treated (P) mice. (b) PIA and Per-induced nanovesicles are internalized. Photomicrographs of $\mathrm{H} 157$ cells incubated for $72 \mathrm{~h}$ with nanovesicle pellet from $1 \mathrm{~h}$ DMSO or PIA, or Per-treated EGFR-GFP H157 donor cells. (c) The $100000 \times g$ media pellet from treated donor cells causes dose-dependent inhibition of proliferation in recipient cells. Shown is the growth of recipient cells compared with untreated control after 72 -h incubation with $100000 \times g$ media pellet from $1 \mathrm{~h}$ PIA or Per-treated EGFR-GFP H157 donor cells. The amount of pellet added to the recipient cells is reflected in the legend. $\times 1$, media pellet from one T75 donor flask, $\times 3$, media pellet from three 775 donor flasks. (d) The $100000 \times$ g media pellet from $1 \mathrm{~h}$ PIA- or Per-treated cells increases ceramide (red color) in recipient cells that is blocked by a SMPD3 inhibitor

These data suggest the toxicity of PIAs or Per might be augmented via nanovesicle transfer and uptake in neighboring cells.

\section{Discussion}

These studies identify sphinomyelinase-dependent generation of ceramide as a class effect of alkylphospholipids and PIAs. PIAs and Per have related chemical structures, as each is comprised of a hydrophobic head group, a phosphate linker and a lipid side chain. Wu et al. ${ }^{12}$ recently showed that SMPD3 possesses an anionic phospholipid-binding site that can stimulate its activity, suggesting that sphingomyelinase activation by PIAs and Per might be direct. In addition, resistance to alkylphospholipids was shown to correlate with levels of sphingomyelin. Van der Luit et al. ${ }^{13}$ showed that downregulation of sphingomyelin synthase abrogates alkylphospholipid-induced cytotoxicity, and a cell line with induced resistance to alkylphospholipids had decreased sphingomyelin and sphingomyelin synthase. As sphingomyelinase cleaves sphingomyelin to generate ceramide, this suggests the cellular response to alkylphospholipids is sensitive to sphingomyelin levels and the conversion of sphingomyelin to ceramide. Of note, several ceramideinducing therapies were shown to synergize with Per. ${ }^{14-16}$

The loss and transfer of cellular components in nanovesicles is consistent with the loss of protein expression by Per that has been reported by Fu et al. ${ }^{17}$ They showed that Per decreased components of the PI3K/mTOR/Akt pathway, such as Akt, raptor, rictor, mtor, p70S6K and 4EBP-1. Although these investigators showed that proteasomal degradation was important, it is possible that a portion of these proteins could be contained in extracellular nanovesicles. Stimulation of nanovesicle release by PIAs or Per did not depend on Akt inhibition, which agrees with previous studies demonstrating PIAs fuction through a distinct mechanism from other PI3K/Akt pathway inhibitors and target other proteins in addition to Akt. ${ }^{2,3,5}$ In contrast to LY, MCD caused similar effects with different kinetics, suggesting that some of the biological activities of these compounds might be due to raft disturbance. Consistent with this, Per was shown to be taken up through, accumulate in, and alter lipid raft 
components. ${ }^{18,19}$ The fact that P5 and Per increased the lipid raft protein Gi $\alpha 2$ in the $100000 \times g$ pellet suggests that PIAs and Per stimulate shedding of raft components in vesicles, a phenomenon that has been described previously. ${ }^{20}$

Cell-derived nanovesicles can originate from a multivesicular endosome (termed exosome) or budding of the plasma membrane. ${ }^{21,22}$ Trajkovic et al. $^{10}$ showed SMPD3-mediated ceramide generation was required for formation of multivesicular endosomes and exosome release. More recently, SMPD1 was shown to regulate cytotoxic granule secretion by $T$ cells and microparticle release from glioma cells. ${ }^{23,24}$ In our study, P5-stimulated a 2-fold induction in ceramide levels, and Per can induce ceramide. ${ }^{25}$ Ceramide induction was important because PIA- and Per-induced nanovesicle release was blocked by pretreatment with a SMPD3 inhibitor, and Per-induced nanovesicle release was partially blocked by an SMPD1 inhibitor. Consistent with the mechanism of vesicle shedding, alkylphospholipids were shown to increase cholesterol efflux. ${ }^{26}$ In addition, treatment with exogenous ceramide increased EGFR, IGFR, CD151 and CD81 in the media. These data suggest that ceramide generation underlies the release of nanovesicles from cancer cells mediated by PIAs and Per.

What are the implications of nanovesicle release for use of these lipid-based Akt inhibitors in cancer therapy? A number of studies have reported detrimental effects of nanovesicles, including tumor promotion, ${ }^{27}$ angiogenesis ${ }^{28,29}$ and immune suppression. Others have shown immune stimulation through exosomal presentation of antigen to dendritic cells, ${ }^{30,31}$ and vaccination with patient-derived exosomes is being investigated as a cancer immunotherapy. ${ }^{32} \mathrm{~A}$ few cytotoxic cancer therapies have been shown to increase nanovesicle release from cancer cells, ${ }^{33,34}$ but their impact on neighboring cells was not assessed. We found that PIA or Per-induced nanovesicles could be taken up by recipient tumor cells, and this mimicked the effect of the original drug treatment, including ceramide induction and increased cytotoxicity. We believe this is the first report of secondary pharmacological effects via drug transfer in nanovesicles. In addition, because membrane vesicles have been isolated from various body fluids, ${ }^{35-38}$ it is possible these could serve as a biomarker of PIA or Per administration. For example, plasma ceramide has been used as a therapeutic biomarker. ${ }^{39}$

In summary, we have identified release of nanovesicles through ceramide induction as a novel class effect of lipidbased Akt inhibitors. Ceramide induction also contributes to P-Akt inhibition and death. The mechanism of targeted agents is important to their clinical development. Although these agents have been primarily thought of as Akt inhibitors, few efforts have been made to assess Akt in patient samples in Per clinical trials. As ceramide is required for cytotoxicity, this may explain why Per has had some success in myeloma, WM and sarcoma clinical trials, yet limited efficacy in other solid tumors. In addition, this study expands the list of proteins that may contribute to PIA or Per-induced cytotoxicity. EGFR and IGF-IR are valid targets in cancer therapy, and developing agents that inhibit oncogenic signaling through receptor tyrosine kinases such as EGFR and IGFR is an intense area of research in oncology. Current strategies include blocking ligand binding, inhibiting kinase activity or inducing receptor degradation. Secretion in cellular nanovesicles may now be added to the potential ways that drugs might inhibit these important growth regulators in tumors.

\section{Materials and Methods}

Cell culture and reagents. $\mathrm{H} 157$ and $\mathrm{H} 460$ human NSCLC cell lines were obtained from NCI/Naval medical oncology (Bethesda, MD, USA), and A549 NSCLC cells were obtained from the ATCC (Manassas, VA, USA). Cell lines were maintained in RPMl 1640 medium containing 5 or $10 \% \mathrm{FBS}$, at $37^{\circ} \mathrm{C}$ in a $5 \% \mathrm{CO}_{2}$ atmosphere. Methyl- $\beta$-cyclodextrin and C2 ceramide were purchased from Sigma (St. Louis, MO, USA). EGF and IGF-I were obtained from R\&D Systems (Minneapolis, MN, USA). GW4869 was obtained from EMD Biosciences (San Diego, CA, USA). Antibodies to EGFR, P-EGFR Y1068, IGF-IR $\beta$, P-IGF-IR Y1131, P-Akt S473, P-Akt T308, Akt, P-p38 T202/Y204, Lamin A/C, Bip, Cox IV and $\beta$-actin were purchased from Cell Signaling (Danvers, MA, USA). CD151, CD81, lamp2 and Gi 2 antibodies were procured from Santa Cruz Biotechnology (Santa Cruz, CA, USA). C6 and C12 ceramide were obtained from Avanti Polar Lipids (Alabaster, AL, USA) and were dissolved in DMSO. Media concentration was performed using a Centricon Ultracel YM-10 filter unit (Millipore, Billerica, MA, USA). Perifosine was obtained from the Developmental Therapeutics Program, $\mathrm{NCl}$ (Frederick, MD, USA). PIAs were synthesized as described previously. ${ }^{40} \mathrm{~V} 5$ tagged plasmids to SMPD3, or SMPD1 were from Y Hannun. Taqman assay for SMPD1 and nontargeting, SMPD1 and SMPD3 siRNA were obtained from Applied Biosystems (Carlsbad, CA, USA).

Immunoblotting. Cells were lysed in $2 X$ LSB as described previously. ${ }^{41}$ Proteins were separated by SDS-PAGE then transferred to nitrocellulose membranes. Membranes were blocked in blocking buffer (1X TBS, 5\% milk and $0.1 \%$ Tween-20) and placed in primary antibody overnight at $4{ }^{\circ} \mathrm{C}$. The following day, membranes were washed in wash buffer $(0.1 \%$ Tween-20, $1 \mathrm{X}$ TBS). Primary antibody was detected using horseradish peroxidase-linked secondary antibodies and visualized with the ECL western blotting detection system (GE Healthcare, Piscatway, $\mathrm{NJ}$, USA). Immunoblot experiments were performed at least three times.

Electron microscopy. The $100000 \times \mathrm{g}$ media pellet from P5- or Per-treated cells was fixed in $4 \%$ paraformaldehyde, and dropped onto formavar carbon-coated grids. The grids were stained with uranyl acetate, and viewed in a Philips CM10 transmission electron microscope (Philips Research, Eindhoven, The Netherlands).

Creation of EGFR-GFP H157 cell line. An eGFP-EGFR plasmid provided by Dr. Alexander Sorkin, University of Colorado was transfected into H157 cells using the Amaxa nucleofection system (Lonza, Basel, Switzerland). Cells with stable integration were selected using G418.

Time-lapse microscopy. H157 cells were plated in Lab-Tek chambered coverglasses (Thermo Fisher Scientific, Waltham, MA, USA) in RPMI + 5\% FBS and allowed to grow overnight. Before imaging, cells were washed with PBS and stained with FM 1-43X (Invitrogen, Carlsbad, CA, USA) in HBSS for 1 min on ice, then washed twice with PBS and fresh RPMI $+0.1 \%$ FBS was added to the chamber. Cells were immediately imaged with a Zeiss NLO microscope (Carl Zeiss Microscopy LLC, Thornwood, NY, USA) for a period of $1 \mathrm{~min}$ before P5 addition. Imaging of the same cells resumed following dosing with P5 after a delay of $\sim 15 \mathrm{~s}$, for a period of $2 \mathrm{~min}$.

Cell culture media concentration and fractionation (nanovesicle isolation). For the initial assessment of cell culture media, media from a T75 flask were concentrated $(\sim 30 \times$ reduction in volume) using a Centricon Ultracel YM-10 filter unit (Millipore), and immunoblot analysis was performed on 1/10 of the remaining media volume. In the remaining experiments, media from either a T75 flask $\left(6 \times 10^{6}\right.$ cells $)$ or three wells of a six-well plate $\left(1.5 \times 10^{6}\right.$ cells $)$ were centrifuged at $300 \times g(10 \mathrm{~min}), 1200 \times g(20 \mathrm{~min})$ and $10000 \times g(30 \mathrm{~min})$ to remove whole cells and debris, then centrifuged at $100000 \times g$ for $60 \mathrm{~min}$ and the pellet was resolubilized in $2 X$ LSB $^{41}$ for immunoblotting or fixed in $4 \%$ neutral buffered formalin for EM studies.

Ceramide assay. The diacylglycerol kinase assay was performed in triplicate as described previously. ${ }^{42}$ 
Sphingomyelinase assay. The Amplex red sphingomyelinase assay was performed according to the manufacturers instructions (Invitrogen).

Pharmacologic treatment. Cells were grown in RPMI $+5 \%$ FBS. Media were removed from cells, washed $\times 1$ with PBS then treated with PIA or Per in $\mathrm{RPMI}+0.1 \% \mathrm{FBS}$ for the indicated times.

Immunofluorescence. Cells were fixed with $4 \%$ paraformaldehyde and stained with an anti-ceramide primary antibody (MID 15B4) from Alexis/Enzo Life Sciences (Plymouth Meeting, PA, USA), and an anti-IGM texas red-conjugated secondary antibody (cat\#1021-07, Southern Biotech, Birmingham, AL, USA).

Transfection experiments. SMPD1 and SMPD3 siRNA or plasmids were delivered to cells using the Amaxa nucleofector system.

In vivo study. Female athymic nude mice were injected subcutaneously with $\mathrm{H} 157$ cells in both rear flanks. When tumors were $\sim 300 \mathrm{~mm}^{3}$, mice were given a single i.p. injection of $90 \mathrm{mg} / \mathrm{kg} \mathrm{P5}$ in $10 \%$ DMSO/saline $/ 0.5 \%$ tween 80 or $40 \mathrm{mg} /$ $\mathrm{kg}$ Per dissolved in saline. After $1 \mathrm{~h}$, mice were anesthetized and cardiac puncture was performed. Blood was collected in BD Microtainer tubes (\#365985, BD, Franklin Lakes, NJ, USA), and plasma was isolated as per the manufacturer's instructions. An equal volume of plasma from each mouse was centrifuged at $300 \times g$ for $10 \mathrm{~min}, 1200 \times g$ for $20 \mathrm{~min}$ and $10000 \times g$ for $30 \mathrm{~min}$. Each sample was diluted 1:10 in PBS and finally spun at $100000 \times g$ for $1 \mathrm{~h}$. The $100000 \times g$ pellet was resolubilized in $2 \times \mathrm{LSB}$ and was run on a $10 \%$ PAGE gel. In vivo experiments were performed twice and were conducted under a protocol approved by the $\mathrm{NCl}$ Animal Care and Use Committee.

Nanovesicle transfer experiments. In all, $6 \times 10^{6}$ EGFR-GFP H157 cells (donor) were treated with DMSO, $10 \mu \mathrm{M}$ P5 or $10 \mu \mathrm{M}$ Per for $1 \mathrm{~h}$. Media were collected and spun at $300 \times g(10 \mathrm{~min}), 1200 \times g(20 \mathrm{~min}), 10000 \times g(30 \mathrm{~min})$ and $100000 \times g(60 \mathrm{~min})$. The final $100000 \times g$ pellet was washed with PBS and recollected by centrifugation for an additional hour. This was added to 20000 recipient H157 (parental) cells growing in an 8-well chamber slide. Recipients were washed five times with RPMI $+5 \%$ FBS after $72 \mathrm{~h}$ and fixed in $4 \%$ paraformaldehyde before DAPI staining and imaging using a Zeiss LSM 510 microscope. Alternatively, recipient cells were fixed and stained with an anticeramide antibody, followed by an anti-IGM texas-red secondary antibody then imaged by fluorescence microscopy.

Statistics. Significant differences between treatment groups were assessed using a non-paired Student's t-test.

\section{Conflict of Interest}

The authors declare no conflict of interest.

Acknowledgements. This research was supported in part by the Intramural Research Program of the NIH, National Cancer Institute, Center for Cancer Research, and in part with federal funds from the National Cancer Institute, National Institutes of Health, under contract NCl Contract NO1-CO-12400. The content of this publication does not necessarily reflect the views or policies of the Department of Health and Human Services, nor does mention of trade names, commercial products or organizations imply endorsement by the US Government.

1. Castillo SS, Brognard J, Petukhov PA, Zhang C, Tsurutani J, Granville CA et al Preferential inhibition of Akt and killing of Akt-dependent cancer cells by rationally designed phosphatidylinositol ether lipid analogues. Cancer Res 2004; 64: 2782-2792.

2. Gills JJ, Castillo SS, Zhang C, Petukhov PA, Memmott RM, Hollingshead M et al Phosphatidylinositol ether lipid analogues that inhibit AKT also independently activate the stress kinase, p38alpha, through MKK3/6-independent and -dependent mechanisms. J Biol Chem 2007; 282: 27020-27029.

3. Memmott RM, Gills JJ, Hollingshead M, Powers MC, Chen Z, Kemp B et al. Phosphatidylinositol ether lipid analogues induce AMP-activated protein kinase-dependent death in LKB1-mutant non small cell lung cancer cells. Cancer Res 2008; 68: 580-588.

4. Zhang C, Elkahloun AG, Liao H, Delaney S, Saber B, Morrow B et al. Expression signatures of the lipid-based akt inhibitors phosphatidylinositol ether lipid analogues in NSCLC cells. Mol Cancer Ther 2011; 10: 1137-1148.
5. Gills JJ, Holbeck S, Hollingshead M, Hewitt SM, Kozikowski AP, Dennis PA. Spectrum of activity and molecular correlates of response to phosphatidylinositol ether lipid analogues, novel lipid-based inhibitors of Akt. Mol Cancer Ther 2006; 5: 713-722.

6. Gills JJ, Dennis PA. Perifosine: update on a novel Akt inhibitor. Curr Oncol Rep 2009; 11: $102-110$

7. Adam RM, Mukhopadhyay NK, Kim J, Di Vizio D, Cinar B, Boucher K et al. Cholesterol sensitivity of endogenous and myristoylated Akt. Cancer Res 2007; 67: 6238-6246

8. Escola JM, Kleijmeer MJ, Stoorvogel W, Griffith JM, Yoshie O, Geuze HJ. Selective enrichment of tetraspan proteins on the internal vesicles of multivesicular endosomes and on exosomes secreted by human B-lymphocytes. J Biol Chem 1998; 273: 20121-20127.

9. Zhuang L, Kim J, Adam RM, Solomon KR, Freeman MR. Cholesterol targeting alters lipid raft composition and cell survival in prostate cancer cells and xenografts. $J$ Clin Invest 2005; 115: 959-968.

10. Trajkovic K, Hsu C, Chiantia S, Rajendran L, Wenzel D, Wieland F et al. Ceramide triggers budding of exosome vesicles into multivesicular endosomes. Science 2008; 319: 1244-1247.

11. Zeidan YH, Jenkins RW, Hannun YA. Remodeling of cellular cytoskeleton by the acid sphingomyelinase/ceramide pathway. J Cell Biol 2008; 181: 335-350.

12. Wu BX, Clarke CJ, Matmati N, Montefusco D, Bartke N, Hannun YA. Identification of novel anionic phospholipid binding domains in neutral sphingomyelinase 2 with selective binding preference. J Biol Chem 2011; 286: 22362-22371.

13. Van der Luit AH, Budde M, Zerp S, Caan W, Klarenbeek JB, Verheij M et al. Resistance to alkyl-lysophospholipid-induced apoptosis due to downregulated sphingomyelin synthase 1 expression with consequent sphingomyelin- and cholesterol-deficiency in lipid rafts. Biochem J 2007; 401: 541-549.

14. David E, Sinha R, Chen J, Sun SY, Kaufman JL, Lonial S. Perifosine synergistically enhances TRAIL-induced myeloma cell apoptosis via up-regulation of death receptors. Clin Cancer Res 2008; 14: 5090-5098.

15. Tazzari PL, Tabellini G, Ricci F, Papa V, Bortul R, Chiarini F et al. Synergistic proapoptotic activity of recombinant TRAIL plus the Akt inhibitor Perifosine in acute myelogenous leukemia cells. Cancer Res 2008; 68: 9394-9403.

16. Sun H, Yu T, Li J. Co-administration of perifosine with paclitaxel synergistically induces apoptosis in ovarian cancer cells: More than just AKT inhibition. Cancer Lett 2011; 310: 118-128

17. Fu L, Kim YA, Wang X, Wu X, Yue $P$, Lonial S et al. Perifosine inhibits mammalian target of rapamycin signaling through facilitating degradation of major components in the mTOR axis and induces autophagy. Cancer Res 2009; 69: 8967-8976.

18. van der Luit AH, Vink SR, Klarenbeek JB, Perrissoud D, Solary E, Verheij M et al. A new class of anticancer alkylphospholipids uses lipid rafts as membrane gateways to induce apoptosis in lymphoma cells. Mol Cancer Ther 2007; 6: 2337-2345.

19. Gajate C, Mollinedo F. Edelfosine and perifosine induce selective apoptosis in multiple myeloma by recruitment of death receptors and downstream signaling molecules into lipid rafts. Blood 2007; 109: 711-719.

20. de Gassart A, Geminard C, Fevrier B, Raposo G, Vidal M. Lipid raft-associated protein sorting in exosomes. Blood 2003; 102: 4336-4344.

21. Schorey JS, Bhatnagar $S$. Exosome function: from tumor immunology to pathogen biology. Traffic 2008; 9: 871-881.

22. Ratajczak J, Wysoczynski M, Hayek F, Janowska-Wieczorek A, Ratajczak MZ. Membranederived microvesicles: important and underappreciated mediators of cell-to-cell communication. Leukemia 2006; 20: 1487-1495.

23. Bianco F, Perrotta C, Novellino L, Francolini M, Riganti L, Menna E et al. Acid sphingomyelinase activity triggers microparticle release from glial cells. EMBO J 2009; 28: 1043-1054

24. Herz J, Pardo J, Kashkar H, Schramm M, Kuzmenkina E, Bos E et al. Acid sphingomyelinase is a key regulator of cytotoxic granule secretion by primary $T$ lymphocytes. Nat Immunol 2009; 10: 761-768.

25. Rahmani M, Reese E, Dai Y, Bauer C, Payne SG, Dent P et al. Coadministration of histone deacetylase inhibitors and perifosine synergistically induces apoptosis in human leukemia cells through Akt and ERK1/2 inactivation and the generation of ceramide and reactive oxygen species. Cancer Res 2005; 65: 2422-2432.

26. Rios-Marco P, Jimenez-Lopez JM, Marco C. Segovia JL, Carrasco MP. Antitumoral alkylphospholipids induce cholesterol efflux from the plasma membrane in HepG2 cells. J Pharmacol Exp Ther 2011; 336: 866-873.

27. Al-Nedawi K, Meehan B, Micallef J, Lhotak V, May L, Guha A et al. Intercellular transfer of the oncogenic receptor EGFRvill by microvesicles derived from tumour cells. Nat Cell Biol 2008; 10: 619-624.

28. Nazarenko I, Rana S, Baumann A, McAlear J, Hellwig A, Trendelenburg M et al. Cell surface tetraspanin Tspan8 contributes to molecular pathways of exosome-induced endothelial cell activation. Cancer Res 2010; 70: 1668-1678.

29. Al-Nedawi K, Meehan B, Kerbel RS, Allison AC, Rak J. Endothelial expression of autocrine VEGF upon the uptake of tumor-derived microvesicles containing oncogenic EGFR. Proc Natl Acad Sci USA 2009; 106: 3794-3799.

30. Wolfers J, Lozier A, Raposo G, Regnault A, Thery C, Masurier C et al. Tumor-derived exosomes are a source of shared tumor rejection antigens for CTL cross-priming. Nat Med 2001; 7: 297-303. 
31. Zitvogel L, Regnault A, Lozier A, Wolfers J, Flament C, Tenza D et al. Eradication of established murine tumors using a novel cell-free vaccine: dendritic cell-derived exosomes. Nat Med 1998; 4: 594-600.

32. Morse MA, Garst J, Osada T, Khan S, Hobeika A, Clay TM et al. A phase I study of dexosome immunotherapy in patients with advanced non-small cell lung cancer J Transl Med 2005; 3: 9.

33. Safaei R, Larson BJ, Cheng TC, Gibson MA, Otani S, Naerdemann W et al. Abnormal lysosomal trafficking and enhanced exosomal export of cisplatin in drug-resistant human ovarian carcinoma cells. Mol Cancer Ther 2005; 4: 1595-1604.

34. Yu X, Harris SL, Levine AJ. The regulation of exosome secretion: a novel function of the p53 protein. Cancer Res 2006; 66: 4795-4801.

35. Zhou H, Pisitkun T, Aponte A, Yuen PS, Hoffert JD, Yasuda H et al. Exosomal Fetuin-A identified by proteomics: a novel urinary biomarker for detecting acute kidney injury. Kidney Int 2006; 70: 1847-1857.

36. Caby MP, Lankar D, Vincendeau-Scherrer C, Raposo G, Bonnerot C. Exosomal-like vesicles are present in human blood plasma. Int Immunol 2005; 17: 879-887.

37. Andre F, Schartz NE, Movassagh M, Flament C, Pautier P, Morice $P$ et al. Malignant effusions and immunogenic tumour-derived exosomes. Lancet 2002; 360: 295-305.

38. Runz S, Keller S, Rupp C, Stoeck A, Issa Y, Koensgen D et al. Malignant ascites-derived exosomes of ovarian carcinoma patients contain CD24 and EpCAM. Gynecol Oncol 2007; 107: $563-571$.
39. Saddoughi SA, Garrett-Mayer E, Chaudhary U, O'Brien PE, Afrin LB, Day TA et al. Results of a phase II trial of gemcitabine plus doxorubicin in patients with recurrent head and neck cancers: serum c18-ceramide as a novel biomarker for monitoring response. Clin Cancer Res 2011; 17: 6097-6105.

40. Kozikowski AP, Sun H, Brognard J, Dennis PA, Novel PI. analogues selectively block activation of the pro-survival serine/threonine kinase Akt. J Am Chem Soc 2003; 125: 1144-1145.

41. Canman CE, Wolff AC, Chen CY, Fornace Jr AJ, Kastan MB. The p53-dependent G1 cell cycle checkpoint pathway and ataxia-telangiectasia. Cancer Res 1994; 54: 5054-5058.

42. Chan C, Goldkorn T. Ceramide path in human lung cell death. Am J Respir Cell Mol Biol 2000; 22: 460-468.

Cell Death and Disease is an open-access journal published by Nature Publishing Group. This work is licensed under the Creative Commons Attribution-NonCommercial-No Derivative Works 3.0 Unported License. To view a copy of this license, visit http://creativecommons.org/licenses/by-nc-nd/3.0/

Supplementary Information accompanies the paper on Cell Death and Disease website (http://www.nature.com/cddis) 